\title{
A TWO-PARAMETER MODEL FOR THE INFRARED/SUBMILLIMETER/RADIO SPECTRAL ENERGY DISTRIBUTIONS OF GALAXIES AND ACTIVE GALACTIC NUCLEI
}

\author{
Daniel A. Dale ${ }^{1}$, George Helou ${ }^{2}$, Georgios E. Magdis ${ }^{3}$, Lee Armus ${ }^{4}$, Tanio Díaz-Santos ${ }^{4}$, And Yong ShI $^{5}$ \\ ${ }^{1}$ Department of Physics and Astronomy, University of Wyoming, Laramie, WY 82071, USA; ddale@uwyo.edu \\ 2 Infrared Processing and Analysis Center, California Institute of Technology, Pasadena, CA 91125, USA \\ ${ }^{3}$ Department of Physics, University of Oxford, Keble Road, Oxford OX1 3RH, UK \\ ${ }_{5}^{4}$ Spitzer Science Center, California Institute of Technology, Pasadena, CA 91125, USA \\ ${ }^{5}$ School of Astronomy and Space Science, Nanjing University, Nanjing 210093, China \\ Received 2013 November 25; accepted 2014 February 6; published 2014 March 6
}

\begin{abstract}
A two-parameter semi-empirical model is presented for the spectral energy distributions of galaxies with contributions to their infrared-submillimeter-radio emission from both star formation and accretion disk-powered activity. This model builds upon a previous one-parameter family of models for star-forming galaxies, and includes an update to the mid-infrared emission using an average template obtained from Spitzer Space Telescope observations of normal galaxies. Star-forming/active galactic nucleus (AGN) diagnostics based on polycyclic aromatic hydrocarbon equivalent widths and broadband infrared colors are presented, and example mid-infrared AGN fractional contributions are estimated from model fits to the Great Observatories All-Sky LIRG Survey sample of nearby U/LIRGS and the Five mJy Unbiased Spitzer Extragalactic Survey sample of $24 \mu \mathrm{m}$ selected sources at redshifts $0 \lesssim z \lesssim 4$
\end{abstract}

Key words: dust, extinction - galaxies: active - galaxies: star formation - infrared: ISM

Online-only material: color figures, extended figures

\section{INTRODUCTION}

There have been many recent developments in modeling galaxy infrared spectral energy distributions (SEDs). Some of these models are quite sophisticated, and when fitted to a galaxy's observed spectrum, their various parameters can yield insight into the physical characteristics of the system (e.g., Silva et al. 1998; Popescu et al. 2000; Gordon et al. 2001; Siebenmorgen \& Krügel 2007; Draine \& Li 2007; da Cunha et al. 2008; Galliano et al. 2008; Groves et al. 2008; Hermelo et al. 2013). Such models are often referred to as "grids" to reflect their multi-dimensional nature. At the other extreme of infrared galaxy spectral models are one-dimensional "templates," typically a suite of synthetic or empirical spectra that essentially rely on a single parameter to characterize a galaxy's infrared spectral shape. For example, Chary \& Elbaz (2001) and Rieke et al. (2009) provide template spectra sequenced according to their bolometric infrared luminosity $L_{\mathrm{TIR}}$. Similarly, Dale \& Helou (2002) use a "single-parameter family" (denoted by their $\alpha_{\mathrm{SF}}$ ) to coherently govern changes across their templates in polycyclic aromatic hydrocarbon (PAH) emission, the peak wavelength of the broad far-infrared bump, and the far-infrared/submillimeter dust emissivity. The work presented in Spoon et al. (2007) represents an example of a spectral set that is intermediate in complexity between grids and templates, whereby the strength of the $9.7 \mu \mathrm{m}$ silicate absorption and the $6.2 \mu \mathrm{m}$ PAH equivalent width form the basis of their two-dimensional system for describing mid-infrared spectra. In deciding which set(s) of models to adopt, the end users ultimately must balance their need for sophisticated interpretation with ease of use. This choice depends on a project's science goals and the richness of the observational data set.

Complicating this choice is the additional issue of infrared emission from active galactic nuclei (AGNs); for many galaxies, a full accounting of their infrared energy budget must include dust for which the heating can be traced to accretion disk-powered luminosity around supermassive black holes, especially for more luminous systems (e.g., Del Moro et al. 2013; Shi et al. 2013; Kirkpatrick et al. 2013). This concern is especially true for interpreting galaxies at higher redshifts $(z \sim 2-3)$, where the fraction of quasars and strong AGN galaxies is higher than at the present epoch (Fan et al. 2001). For example, work by Goto et al. (2010) and Fu et al. (2010) suggest that a significant portion of the evolution with redshift in the cosmic infrared luminosity function can be attributed to the increased fraction of AGNs in the overall galaxy population at higher redshifts. Thus, ideally each set of infrared galaxy SED models would have a convenient methodology for consideration of AGN contributions. Some models do incorporate dust emission from both AGNs and star formation (Siebenmorgen \& Krügel 2007; Rieke et al. 2009; Berta et al. 2013), but most do not. However, the clear challenge in this arena is the paucity of robust multi-wavelength AGN databases, particularly databases where it is clear that the majority of the mid-infrared emission does indeed come from the AGN and not from star-forming regions. Fortunately, recent progress in robust panchromatic AGN data sets present opportunities to remedy this limitation in galaxy spectral modeling (e.g., Richards et al. 2006; Shang et al. 2011; Mullaney et al. 2011; Shi et al. 2013).

We report here efforts to update the infrared/submillimeter/ radio SED models from Dale \& Helou (2002) in two important ways. First, we update the mid-infrared portion of these starforming models, which was originally based on ISOPHOT data from the Infrared Space Observatory (ISO), using results from the Spitzer Space Telescope. The main improvement resulting from this modification is the inclusion of the prominent $17 \mu \mathrm{m}$ PAH complex, which can produce up to $10 \%$ of the total PAH emission. Second, we add another spectral component that represents emission from AGNs; the models in Dale \& Helou (2002) were purely for star-forming systems. For 

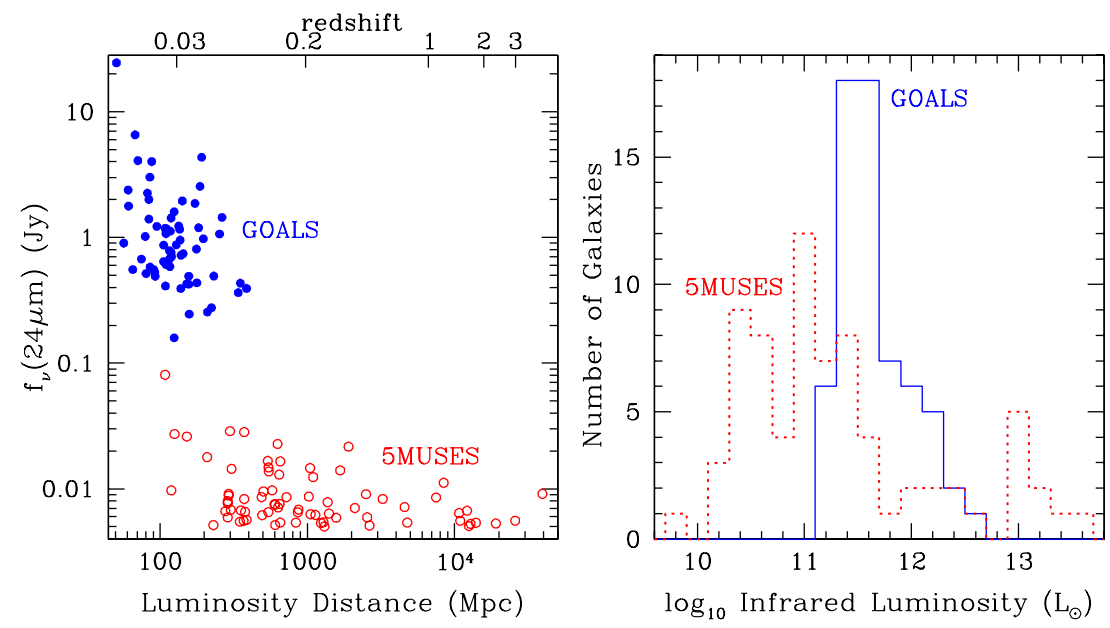

Figure 1. (Subsets of the) two comparison samples used in this work. The subset of the 5MUSES sample (Wu et al. 2010) is the 74 systems with available Spitzer and Herschel/SPIRE photometry (Magdis et al. 2013) and the subset of the GOALS sample (Armus et al. 2009) is the 64 targets with Spitzer photometry (U et al. 2012). The luminosities in the right-hand panel come from U et al. (2012; GOALS) and this work (5MUSES).

(A color version of this figure is available in the online journal.)

this AGN component, a panchromatic database of unobscured Type 1 quasars is employed. We test this new model using data from the Spitzer Space Telescope and Herschel Space Observatory and the 5MUSES (Wu et al. 2010) and GOALS (Armus et al. 2009) surveys, surveys for which AGN percentages have been independently estimated from infrared data (Petric et al. 2011; Wu et al. 2011). In the process, we show these models are applicable to the luminous infrared galaxy (LIRG) and ultraluminous infrared galaxy (ULIRG) regimes $\left(L_{\mathrm{TIR}}>\right.$ $10^{11} L_{\odot}$ and $L_{\mathrm{TIR}}>10^{12} L_{\odot}$, respectively); the models were originally developed using only "normal" star-forming galaxies $\left(L_{\mathrm{TIR}} \lesssim 10^{10} L_{\odot}\right)$.

In Section 2 we review the two galaxy samples against which the updated models are tested. Section 3 describes how the templates are updated using recent AGN and star-forming galaxy databases, and Section 4 presents the results from this work. The final section summarizes our findings.

\section{SAMPLES FOR TESTING THE MODEL}

Two galaxy surveys are used to check the utility of the SED models described below (see Figure 1). The first survey is the Five mJy Unbiased Spitzer Extragalactic Survey (5MUSES; Wu et al. 2010), a $24 \mu \mathrm{m}$ selected sample of 330 galaxies spanning redshifts $0 \lesssim z \lesssim 4$ for which Spitzer Infrared Spectrograph (IRS) low-resolution spectra (5-35 $\mu \mathrm{m})$ were obtained (highresolution IRS spectroscopy was also obtained for a subset of the sample). In addition to the extant Spitzer IRAC and MIPS photometry for 5MUSES that is available from the SWIRE (Lonsdale et al. 2003) and First Look Surveys (Fadda et al. 2006; Frayer et al. 2006), we also have new Herschel 250, 350, and $500 \mu \mathrm{m}$ SPIRE fluxes (Magdis et al. 2013). These Herschel data were taken as part of the HerMES project (Oliver et al. 2010 ), and probe to a 50\% source recovery limit of 12-30 mJy. Overall, secure SPIRE photometry for all three passbands exists for 74 5MUSES targets.

The second comparative sample stems from the Great Observatories All-Sky LIRG Survey (GOALS; Armus et al. 2009). This survey includes deep Spitzer IRS spectroscopy for 202 nearby LIRGs and ULIRGs covering a redshift range of $0 \lesssim$ $z \lesssim 0.09$. Broadband infrared data from Spitzer IRAC 3.6/4.5/ 5.8/8.0 $\mu \mathrm{m}$ and Spitzer MIPS 24/70/160 $\mu \mathrm{m}$ also exist for
GOALS, and we utilize the published photometry for a subset of 64 sources ( $U$ et al. 2012). Using the mid-infrared continuum spectral diagnostics developed in Laurent et al. (2000), the typical AGN fractional contribution to the mid-infrared energy budget in GOALS sources is 15\% (Petric et al. 2011).

\section{THE UPDATED SPECTRAL ENERGY DISTRIBUTION MODELS}

In the original construction of these templates (Dale et al. 2001; Dale \& Helou 2002), a series of "local" SEDs were created to represent the emission from dust exposed to a wide range of heating intensities $0.3 \leqslant U \leqslant 10^{5}$ where $U=1$ corresponds to the local interstellar radiation field in the solar neighborhood. A power-law combination of these local curves can effectively mimic the spatially integrated ("global") dust emission, i.e.,

$$
d M_{\mathrm{d}} \propto U^{-\alpha_{\mathrm{SF}}} d U,
$$

where $M_{\mathrm{d}}$ is the dust mass heated by a radiation field at intensity $U$ and the exponent $\alpha_{\mathrm{SF}}$ represents the relative contributions of the different local SEDs.

These templates were built on the framework of Désert et al. (1990) and are comprised of emission from stochastically heated PAHs, emission from semi-stochastically heated very small grains, and thermal emission from large dust grains. Various modifications based on observations of star-forming galaxies were made by Dale and collaborators to the Désert et al. (1990) framework, including the insertion of an empirical PAH spectrum, the incorporation of a wavelength-dependent far-infrared/submillimeter dust emissivity, and the extension of their modeling to radiation fields $U>10^{3}$.

The average 2.5-11.5 $\mu \mathrm{m}$ mid-infrared spectrum of $\mathrm{Lu}$ et al. (2003) was used to replace the PAH spectrum of Désert et al. (1990). This SED was derived from the average of 40 normal star-forming disk galaxies from the ISO Key Project on Normal Galaxies (Dale et al. 2000). Though this ISO spectrum represented a step forward in infrared SED modeling at the time, a description here of some of its features and limitations is warranted. Those data were taken with the ISOPHOT instrument aboard ISO, an instrument which had a $24^{\prime \prime} \times 24^{\prime \prime}$ field of view, resulting in relatively large $\sim 4 \mathrm{kpc}$ sizescales over which the galaxies were sampled; the average ISOPHOT spectra 

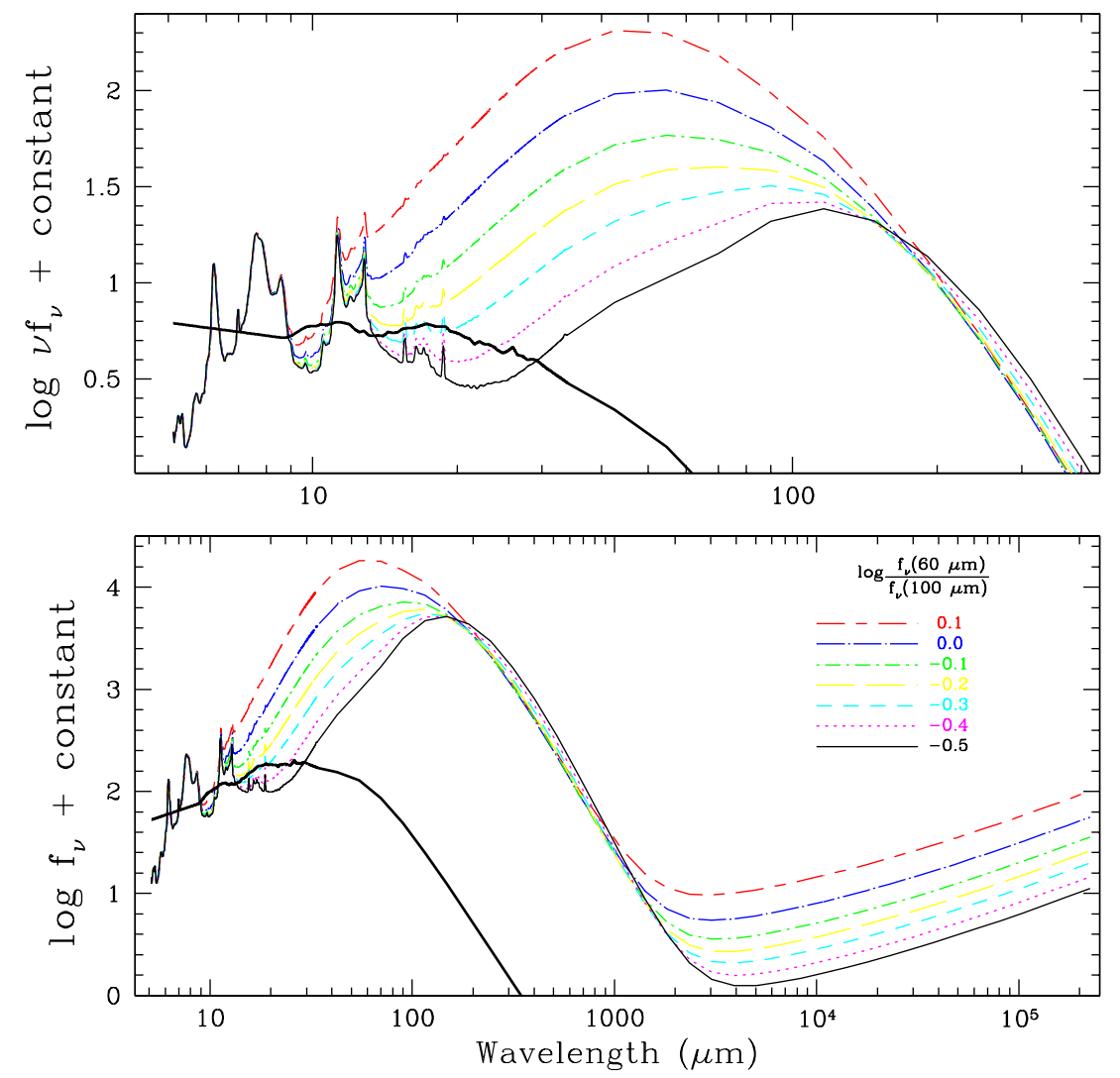

Figure 2. Collection of seven pure star-forming model spectral energy distributions along with that for a pure AGN. The star-forming spectra are essentially the suite of curves presented in Dale \& Helou (2002), but with the ISOPHOT mid-infrared template replaced by the star-forming template of Spoon et al. (2007, their "1C" curve). The different star-forming curves portrayed here represent different $\alpha_{\mathrm{SF}}$ values. The AGN spectrum derives from the median quasar spectral energy distribution of Shi et al. (2013); see Section 3.2.

(A color version of this figure is available in the online journal.)

undoubtedly contained contributions from a wide range of environments (e.g., H II regions; photo-dissociation regions, molecular clouds, etc.), a feature that may not be desirable for certain modeling applications. A significant limitation to the Lu et al. (2003) spectrum is that it is restricted to wavelengths $2.5 \mu \mathrm{m} \leqslant \lambda \leqslant 11.5 \mu \mathrm{m}$ with a gap in coverage between 4.8 and $5.8 \mu \mathrm{m}$. The wavelength gap was bridged with a simple linear interpolation of the average spectrum. Finally, the $11.5 \mu \mathrm{m}$ cut-off to the red end of the ISOPHOT spectrum unfortunately resulted in a truncated tracing of the $11.3 \mu \mathrm{m}$ PAH feature and the omission of the $12.7 \mu \mathrm{m}$ PAH emission feature and those at any longer wavelengths. This latter limitation was partly remedied in Dale et al. (2001) by a schematic extension to $15 \mu \mathrm{m}$ that was guided by ISOCAM circular variable filter observations.

\subsection{Modifications to the Star-forming Templates}

For the updated mid-infrared spectrum we adopt the 5-34 $\mu \mathrm{m}$ "pure" star-forming curve from (Spoon et al. 2007, their spectrum "1C"), who utilized the Spitzer archives to analyze a sequence of mid-infrared spectral shapes among AGNs, ULIRGs, and star-forming galaxies. Important benefits to updating the mid-infrared with Spitzer data are the inclusion of prominent fine-structure lines (e.g., [Ne III] $15.6 \mu \mathrm{m}$, [S III] $18.7 \mu \mathrm{m}$, and [S III] $33.5 \mu \mathrm{m}$ ) and the $17 \mu \mathrm{m}$ PAH complex, the latter of which accounts for up to $10 \%$ of the total PAH emission in normal star-forming galaxies (see Figure 2 of this work and Table 7 of Smith et al. 2007). As was done in Dale et al. (2001), we scale the empirical mid-infrared spectrum to the amplitude of the Désert et al. (1990) PAH templates via integrating over the $12 \mu \mathrm{m} I R A S$ filter. The shape of the mid-infrared continuum beyond $15 \mu \mathrm{m}$ was also fixed to that of the Désert et al. (1990) PAH templates. Besides these modifications to the mid-infrared spectrum, the star-forming templates are otherwise unchanged. We continue to utilize a single-parameter family (i.e., $\alpha_{\mathrm{SF}}$ ) to describe the full range of $\mathrm{PAH} /$ very small grain/large grain and overall spectral shapes for normal star-forming galaxies. Moreover, it should be noted that we continue to assume optically thin infrared emission, and thus do not include in our model any absorption features such as the $9.7 \mu \mathrm{m}$ silicate trough found in many ULIRGs (e.g., Armus et al. 2007). While this simplification will fail to appropriately characterize all the nuances in mid-infrared spectra for samples specifically selected to be infrared-luminous (e.g., GOALS; Stierwalt et al. 2013), there are very few deeply obscured systems in infrared flux-limited surveys like 5MUSES (Wu et al. 2010).

\subsection{Addition of an AGN Template}

While the star-forming templates themselves from Dale \& Helou (2002) are only slightly modified, we introduce here a fundamental addition to the templates by incorporating a second parameter, one that accounts for accretion disk-powered infrared luminosity.

Until recently, the state-of-the-art in panchromatic AGN SEDs was still the pioneering work of Elvis et al. (1994), who studied 47 non-blazar quasars from the radio through $10 \mathrm{keV}$ 


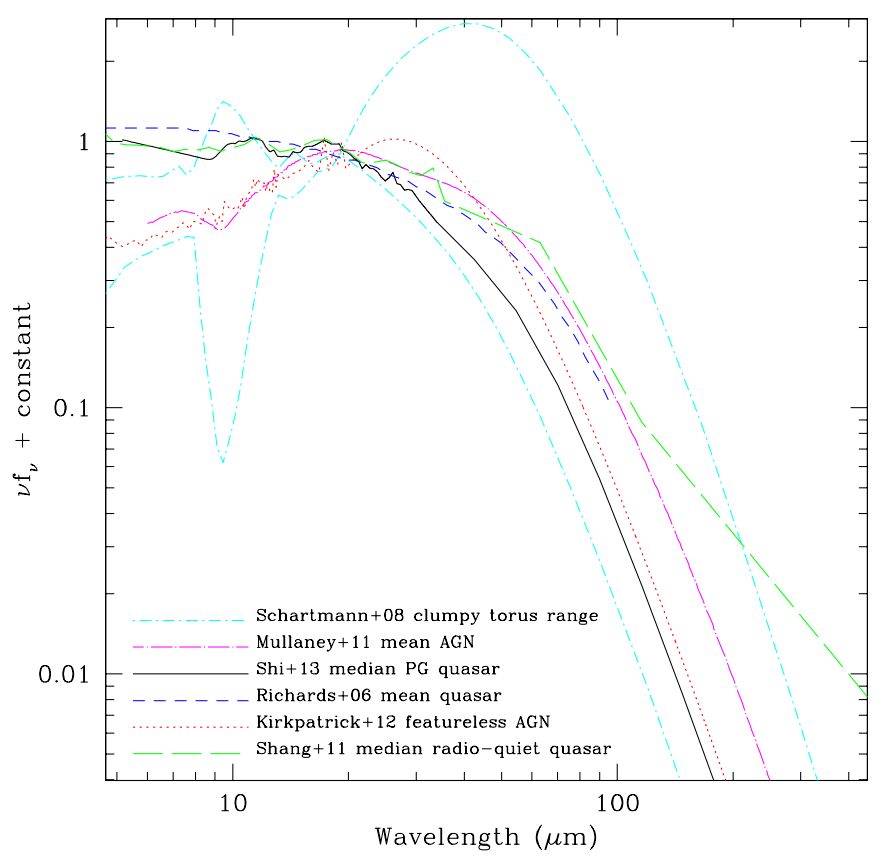

Figure 3. Compilation of several infrared AGN/quasar templates/models from the literature (Richards et al. 2006; Schartmann et al. 2008; Mullaney et al. 2011; Shang et al. 2011; Kirkpatrick et al. 2012; Shi et al. 2013).

(A color version of this figure is available in the online journal.)

X-rays. However, the recent influx of large multi-wavelength databases has allowed for more complete reconstructions of AGN SEDs. For example, in 2011 Shang and collaborators updated the Elvis et al. (1994) work using data from 85 nonblazar quasars. The data involved in their analysis include X-ray, far- and near-ultraviolet, optical, near-, mid-, and farinfrared, and radio spectroscopy and/or photometry. Shi et al. (2013) utilize Spitzer infrared spectral (5-30 $\mu \mathrm{m})$ and imaging (24, 70, and $160 \mu \mathrm{m})$ data from all Palomar-Green quasars to generate a median mid-infrared spectrum similar to that of Shang et al. (2011). These quasars are UV selected and thus are minimally obscured Type 1 AGNs. Figure 3 provides a comparison of several AGN/quasar infrared templates available from the literature as well as the maximum and minimum curves based on the clumpy torus models of Schartmann et al. (2008). We adopt here the median spectrum of Shi et al. (2013) since they have carefully attempted to remove any star formationrelated contributions from the host galaxies, including forcing the template beyond $70 \mu \mathrm{m}$ to drop like a blackbody $\left(v f_{v} \propto \lambda^{-4}\right.$; see their Figure 3). Several features are evident in this median quasar spectrum of Shi et al. (2013), including the broad silicate emission features near 10 and $18 \mu \mathrm{m}$ and the [O IV] $25.9 \mu \mathrm{m}$ fine-structure lines that are seen in many AGNs (Hao et al. 2005; Armus et al. 2007).

Figure 2 displays this median quasar SED of Shi et al. (2013) in addition to a suite of normal star-forming galaxy curves spanning a range in $\alpha_{\mathrm{SF}}$. To simulate the spectral appearance of a source for which the emission has contributions from both an AGN and normal star formation, we employ linear mixing over the 5-20 $\mu \mathrm{m}$ wavelength range. For this work we have developed mixed combinations for 5-20 $\mu \mathrm{m}$ AGN fractions running from $0 \%$ to $100 \%$, spaced at $5 \%$ intervals. $^{6}$ Figure 4 shows how the resulting infrared-radio SEDs appear for a variety of combinations of AGN and star-forming emission.

\footnotetext{
6 Available at physics.uwyo.edu/ ddale/research/seds/seds.html
}

\section{RESULTS}

\subsection{Model Color Distributions}

To date most efforts to disentangle infrared emission from AGNs and star formation have focused on utilizing mid-infrared continuum data sets (e.g., Laurent et al. 2000; Murphy et al. 2009; Mullaney et al. 2011; Wu et al. 2011) or a combination of the mid-infrared continuum plus mid-infrared fine-structure lines (e.g., Genzel et al. 1998; Peeters et al. 2004; Armus et al. 2007; Dale et al. 2009). A common complementary technique for identifying AGN contributions, especially useful when midinfrared spectral data are unavailable, involves combinations of flux ratios that utilize data from three or four broadband filters (e.g., Lacy et al. 2004; Stern et al. 2005; Yan et al. 2013; Kirkpatrick et al. 2013; Mendez et al. 2013). Figure 5 shows how the various combinations of the star-forming and AGN templates appear in two different infrared color-color diagrams (assuming rest wavelengths). Using such continuum diagnostics, one can estimate both the AGN fractional contribution as well as the characteristics of the star-forming portion of the galaxy, e.g., dust temperature. The colors for local actively star-forming galaxies (filled squares) shown in Figure 5 come from Siebenmorgen \& Krügel (2007), the colors for normal star-forming galaxies (open circles) are from Dale et al. (2012), and the colors for the local AGN M87 (filled triangle) are from NED; the $f_{v}(70 \mu \mathrm{m}) / f_{v}(500 \mu \mathrm{m})$ color for M87 falls below the displayed range since the $500 \mu \mathrm{m}$ flux is overwhelmed by synchrotron radiation (Baes et al. 2010). While some (SINGS/KINGFISH) galaxies from Dale et al. (2012) have nuclei that are distinguished by Seyfert or LINER characteristics, very few have their global luminosity dominated by an active nucleus (Moustakas et al. 2010). Note that our color-color analysis does not to extend to wavelengths shorter than $8 \mu \mathrm{m}$ and thus cannot be directly compared to Spitzer IRAC color-color analyses (e.g., Lacy et al. 2004; Stern et al. 2005). This restriction is by design: the Spoon et al. (2007) star-forming template begins at $5 \mu \mathrm{m}$, a feature which conveniently minimizes complications arising from stellar emission.

\subsection{PAH Equivalent Width Distributions}

The strengths of various PAH features have been widely used to diagnose the main power source of a galaxy (e.g., Genzel et al. 1998; Laurent et al. 2000; Armus et al. 2007; Smith et al. 2007; Spoon et al. 2007; Dale et al. 2009; Hernán-Caballero et al. 2009; Diamond-Stanic \& Rieke 2010; Wu et al. 2010; Shang et al. 2011). These studies suggest that EW (PAH $6.2 \mu \mathrm{m}) \approx 0.2 \mu \mathrm{m}$ is an approximate delineation between sources predominantly powered by AGNs and those mostly powered by star formation. Figure 6 shows how the PAH $(6.2 \mu \mathrm{m})$ equivalent widths for our models depend on far-infrared color. The different curves show the trends for a variety of AGN fractions; the $6.2 \mu \mathrm{m}$ equivalent width for the Spoon et al. (2007) pure star-forming curve used here is $\sim 0.5 \mu \mathrm{m}$. As can be seen from the figure, larger AGN fractions correspond to lower equivalent width, attributable to the fact that the adopted AGN template is essentially devoid of PAH emission features. Moreover, each trend of connected points in Figure 6 dips to lower equivalent widths at warmer far-infrared colors, a feature that complicates using the $6.2 \mu \mathrm{m}$ equivalent width as a pure AGN/star-forming diagnostic. This effect of diminished PAH strength (equivalent width) as a function of star formation activity level is weakly built in to the star-forming models (see Figure 6 of Dale et al. 2001), echoing the results of diminished PAH emission for regions 

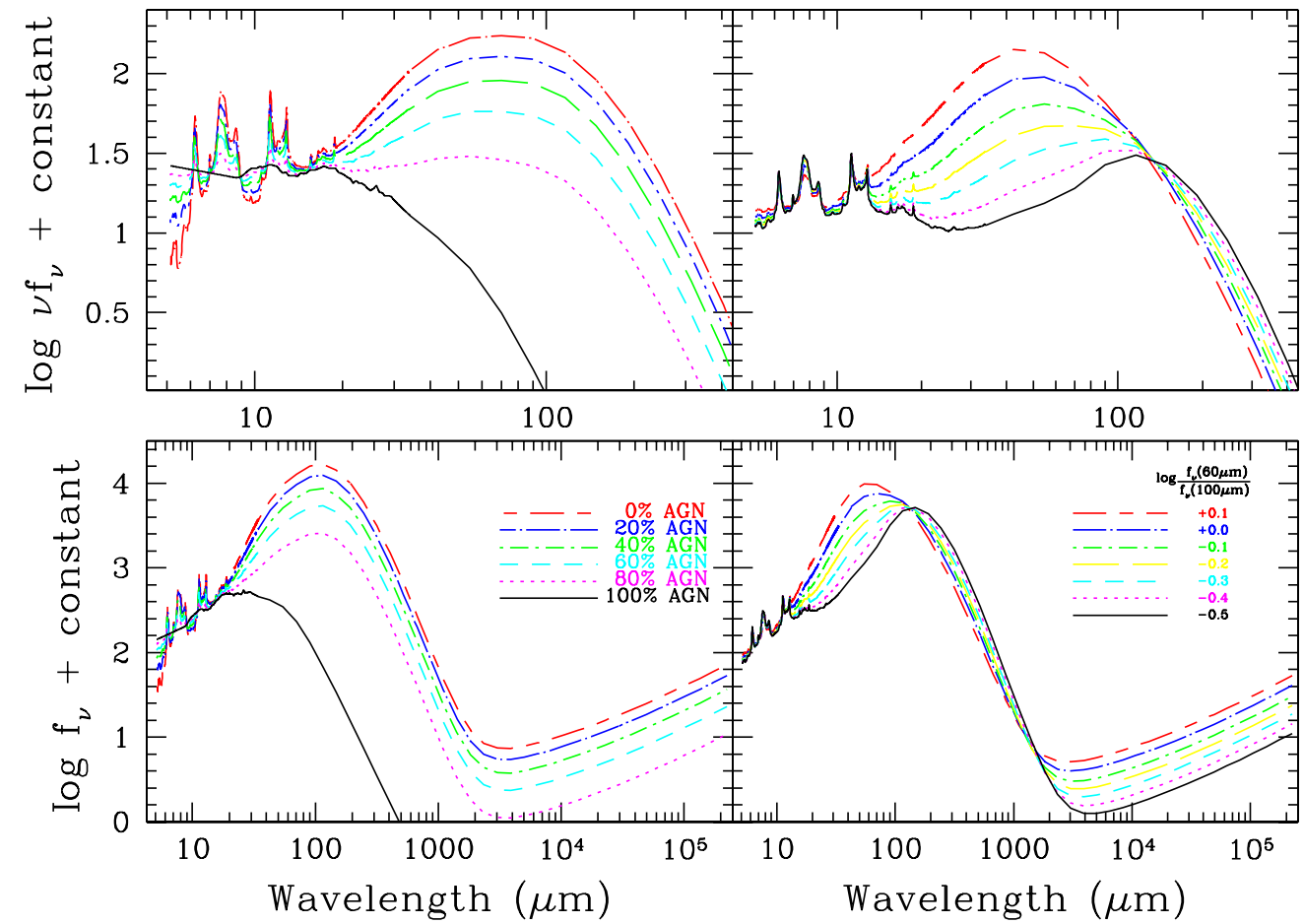

Figure 4. Left: the model curves that result from combining a fixed star-forming template $\left(\alpha_{\mathrm{SF}}=-0.2\right)$ with a variable contribution to the 5-20 $\mu \mathrm{m}$ mid-infrared emission from the radio-quiet quasar curve in Figure 2. Right: the model curves that result from equally combining star-forming templates with the quasar curve. "Equal" implies a 50\% contribution to the emission over 5-20 $\mu \mathrm{m}$.

(A color version of this figure is available in the online journal.)

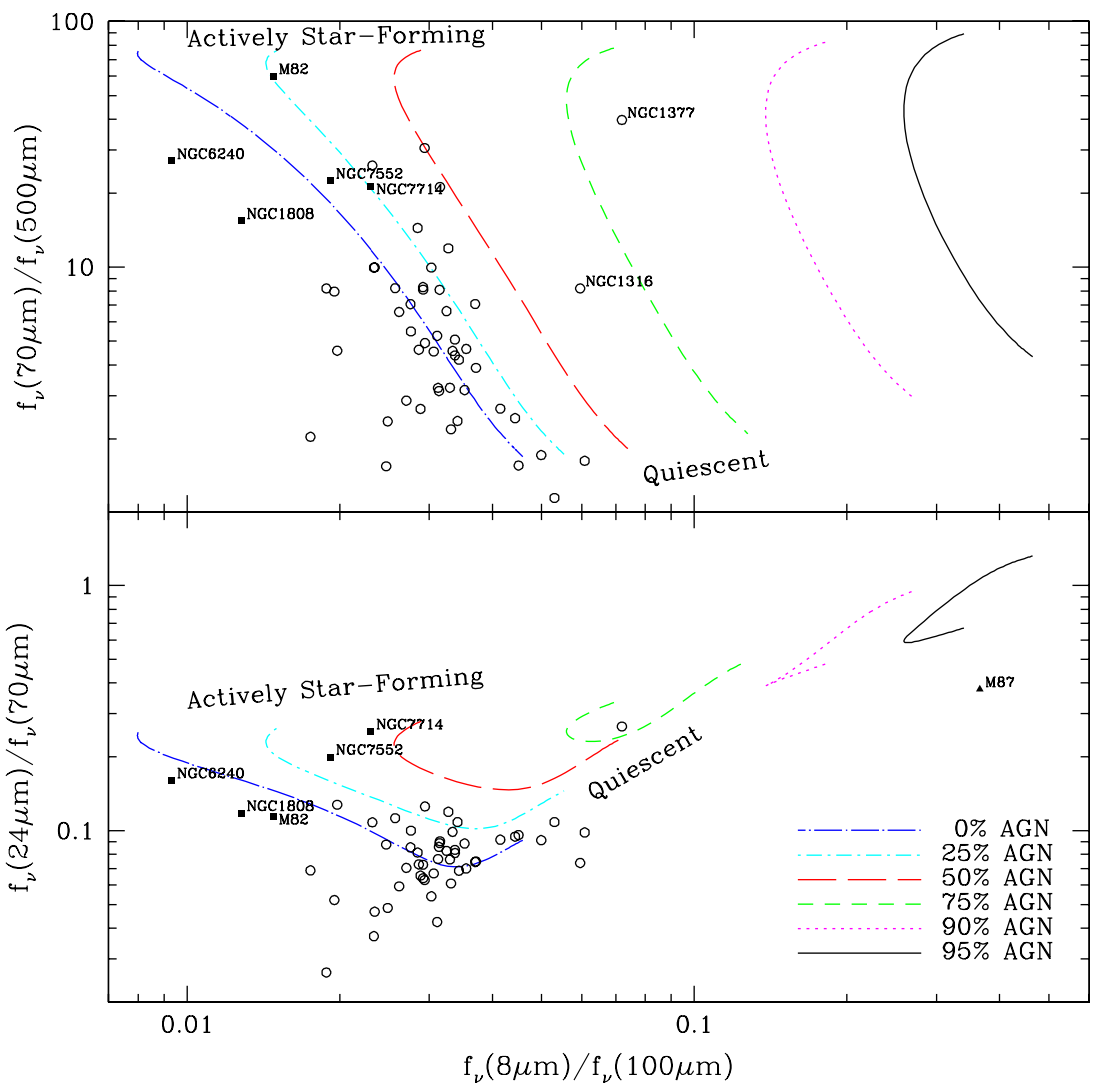

Figure 5. Rest-frame color-color diagrams for the joint AGN-star-forming spectral energy distribution models. The colors for actively star-forming galaxies (filled squares) come from Siebenmorgen \& Krügel (2007), the colors for normal star-forming galaxies (open circles) are from Dale et al. (2012), and the colors for the local AGN M87 (filled triangle) are from the NASA/IPAC Extragalactic Database. The range of model colors displayed for a given mid-infrared AGN percentage represents the diversity of colors in the star-forming templates; the color spread for a single AGN percentage indicates the impact of varying $\alpha_{\mathrm{SF}}$. Note that the terms "actively star-forming" and "quiescent" can be directly tied to the average dust temperature, which in turn is parameterized by $\alpha_{\mathrm{SF}}(\mathrm{Chapman}$ et al. 2003).

(A color version of this figure is available in the online journal.) 


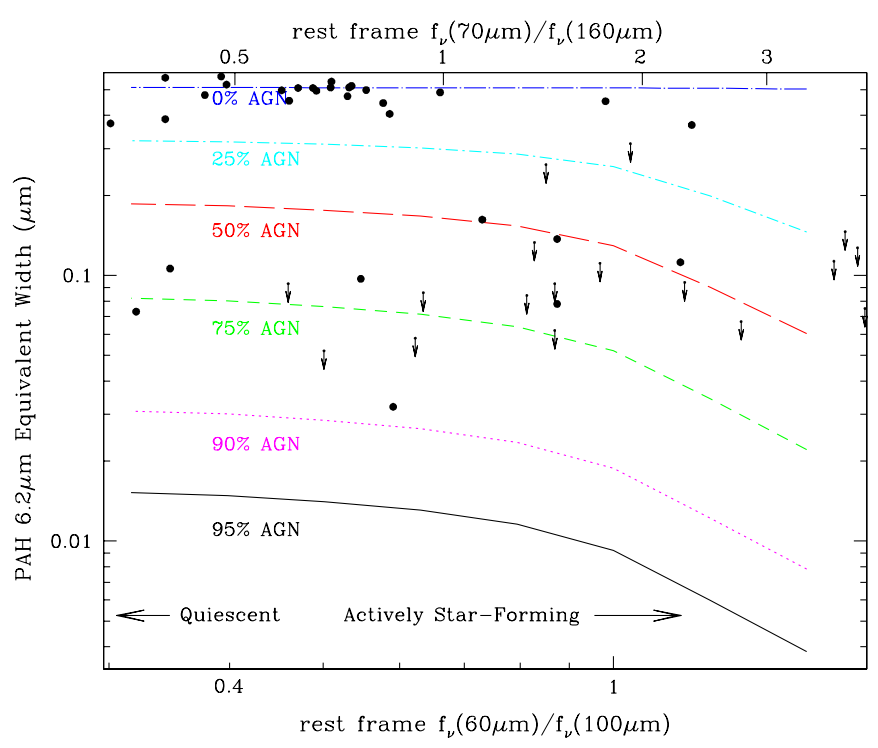

Figure 6. PAH $6.2 \mu \mathrm{m}$ equivalent width as a function of far-infrared color. The different curves indicate the trends for varying mid-infrared fractional levels of AGNs. The data points represent the 5MUSES survey; the colors are based on the fits shown in Figure 7.

(A color version of this figure is available in the online journal.)

permeated by hard radiation fields (Madden et al. 2006). The effect is accentuated when an AGN continuum is added to the mid-infrared. In addition, even "pure" star-forming galaxies exhibit a significant dispersion in the equivalent width of PAH features (e.g., 0.2 dex at $6.2 \mu \mathrm{m}$; Wu et al. 2010), a dispersion that these simple models do not incorporate. However, in agreement with the references listed above, this plot shows that $\mathrm{EW}(\mathrm{PAH} 6.2 \mu \mathrm{m}) \approx 0.2-0.3 \mu \mathrm{m}$ could roughly be used as a demarcation between sources powered by star formation and AGN activity.

\subsection{Spectral Energy Distribution Fits}

Figure 7 shows the best fits of the AGN/star-forming curves to the subset of 5MUSES sources that have Herschel SPIRE data available; Figure 8 provides similar displays for fits to GOALS sources. The fits are carried out via a $\chi^{2}$ minimization using infrared colors:

$$
\chi^{2}=\sum_{i, j<i} \frac{\left(\log \frac{f_{v, i}^{\text {obs }}}{f_{v, j}^{\text {obs }}}-\log \frac{f_{v, i}^{\text {model }}}{f_{v, j}^{\text {model }}}\right)^{2}}{\left(\sigma_{i, j}^{\text {obs }}\right)^{2}},
$$

where $\log f_{v, i}^{\text {obs }} / f_{v, j}^{\text {obs }}$ and $\log f_{v, i}^{\text {model }} / f_{v, j}^{\text {model }}$ are respectively the observed and model colors involving bandpasses $i$ and $j$, and $\sigma_{b}^{\text {obs }}$ is the uncertainty in the observed color. The model colors are obtained after convolving the model with the appropriate filter bandpasses. The colors used in the 5MUSES fits involve all possible combinations of the Spitzer IRAC 5.8/8.0 $\mu \mathrm{m}$, Spitzer MIPS 24/70/160 $\mu \mathrm{m}$, and Herschel SPIRE 250/350/500 $\mu \mathrm{m}$ flux densities, except for the minority of higher redshift targets for which the central wavelengths of certain shorter-wavelength filter bandpasses correspond to rest wavelengths shorter than $5 \mu \mathrm{m}$. For example, the 5.8 and $8.0 \mu \mathrm{m}$ data are not used in the fit for 5MUSES-130 at $z=1.814$ and the 5.8, 8.0, and $24 \mu \mathrm{m}$ data are not used in the fit for 5MUSES-312 at $z=4.270$. The colors used in the GOALS fits involve Spitzer
IRAC 5.8/8.0 $\mu \mathrm{m}$, Spitzer MIPS 24/70/160 $\mu \mathrm{m}$, and IRAS 12/ $25 / 60 / 100 \mu \mathrm{m}$ data, where available.

The values of $\alpha_{\mathrm{SF}}$ and the mid-infrared AGN percentage found in each subpanel of Figures 7 and 8 correspond to the median value obtained after carrying out 1000 Monte Carlo simulations of each fit. For each Monte Carlo simulation, a random (Gaussian deviate) flux offset, scaled according to the measured uncertainty, was added to each flux. Typical uncertainties are a few to several percent, with a slight increase as the number of available fluxes decreases. The use of a different AGN template such as the one from Kirkpatrick et al. (2012) displayed in Figure 3, where the spectrum rises more steeply in the mid-infrared, results in mid-infrared fractions that differ by $2 \%-3 \%$ (with a scatter of a few to several percent) compared to those resulting from the use of the Shi et al. (2013) quasar template (see the bottom row of Figure 9).

\subsection{Comparison with Other AGN Fractional Estimates}

The mid-infrared AGN fractions for galaxies from the 5MUSES and GOALS surveys are provided within the subpanels of Figures 7 and 8. Sixty-eight (eighty-nine) percent of the 5MUSES (GOALS) subsample studied here have mid-infrared AGN fractions less than 50\%, and the mid-infrared AGN fraction unsurprisingly scales strongly with luminosity (see Section 4.5). These 5.8-500 $\mu \mathrm{m}$ SED-fitting-based mid-infrared AGN fractional estimates for both the 5MUSES survey and the GOALS survey can be compared to estimates found in the literature (see the top row of Figure 9). The 5MUSES mid-infrared AGN fractions are compared to those from Wu et al. (2011), who utilize the 5-35 $\mu \mathrm{m}$ continuum data and various templates of AGN and star-forming systems. The GOALS mid-infrared AGN fractions are compared to those from Petric et al. (2011), who employ the mid-infrared spectroscopic diagnostic of Laurent et al. (2000) which compares the slope of the 5-15 $\mu \mathrm{m}$ continuum and the equivalent width of the $6.2 \mu \mathrm{m}$ PAH feature to templates of pure AGN, star formation, and photo-dissociation regions. The mean differences between our mid-infrared AGN percentages and those from the literature are relatively close to zero- $13 \%$ for 5 MUSES and $2 \%$ for GOALS - and the standard deviations in the differences are 18\% (5MUSES) and $15 \%$ (GOALS). In an attempt to understand the systematic 13\% difference for the 5MUSES sample, we tried various combinations of including/excluding photometry from certain filters in the fits, but every combination tested yielded similar results. Thus, the difference is more fundamental than merely the difference in wavelength ranges utilized in our fits and those of $\mathrm{Wu}$ et al. (2011).

For the comparison involving GOALS, a (nonparametric) Spearman rank correlation test yields a correlation coefficient of 0.43 for the 58 targets for which reliable mid-infrared AGN fractions are available from both Petric et al. (2011) and this work. For the 5MUSES subsample of 74 targets studied here, the correlation coefficient is 0.68 . Thus, for both samples there is less than a $1 \%$ probability that the correlation occurred purely through chance.

\subsection{Total Infrared Estimators and AGN Fraction}

Simple prescriptions for estimating the total luminosity over $5-1100 \mu \mathrm{m}^{7}$ can be obtained from linear combinations of

\footnotetext{
7 The TIR wavelength range defined here is slightly different from the 3-1100 $\mu \mathrm{m}$ wavelength range presented in Dale \& Helou (2002) in order to minimize any influence from stellar emission in observed SEDs.
} 


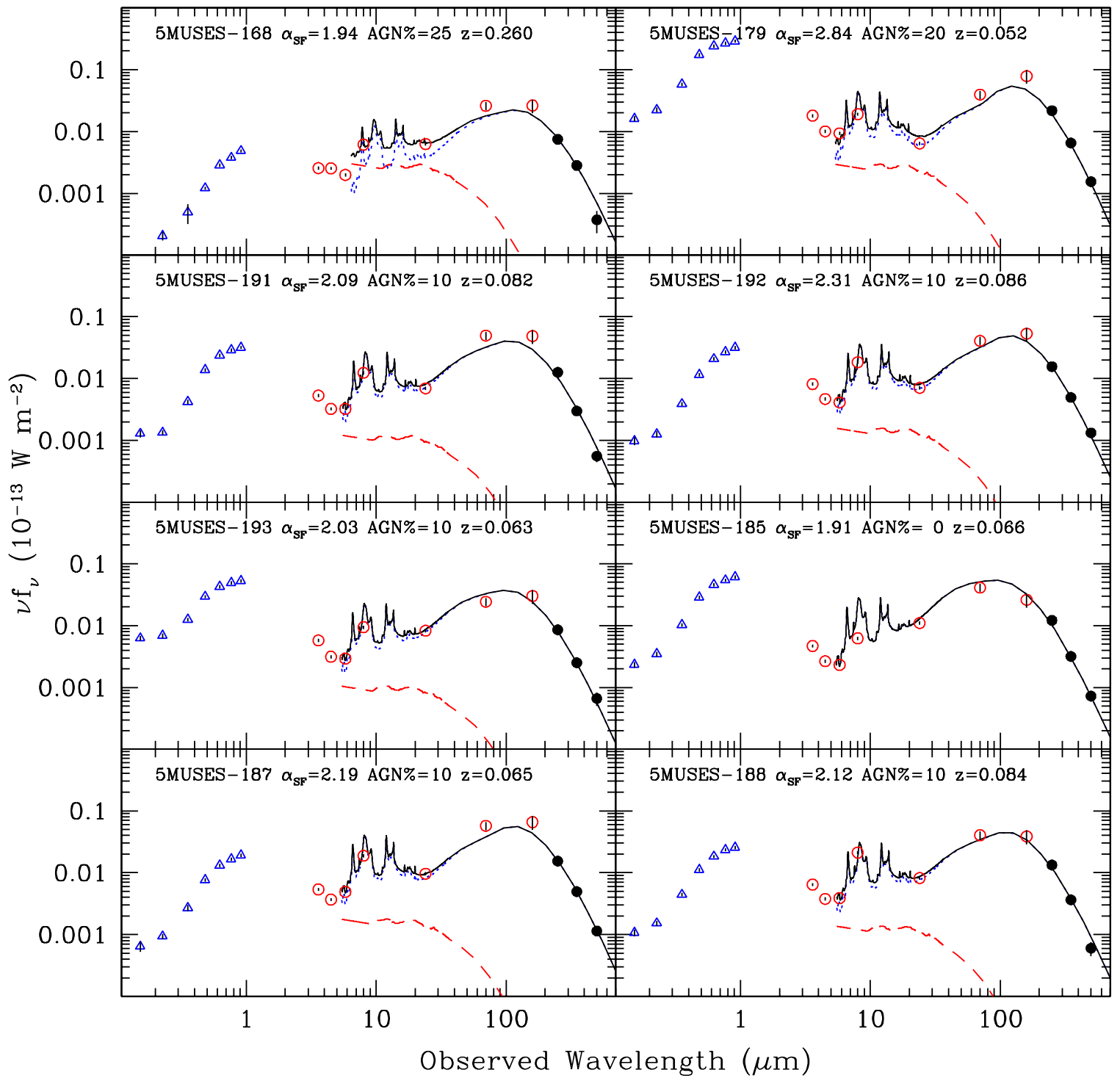

Figure 7. Globally integrated infrared/submillimeter spectral energy distributions for the 5MUSES sample of $24 \mu \mathrm{m}$ selected galaxies (Wu et al. 2010; Magdis et al. 2013), sorted by right ascension. Open circles represent Spitzer data, filled circles are from the Herschel Space Observatory, and open triangles stem from Galaxy Evolution Explorer (GALEX) and the Sloan Digital Sky Survey. The dotted and dashed lines respectively trace the fitted star-forming and AGN components; the sum of the two components (solid line) is normalized to the Spitzer/MIPS and Herschel/SPIRE data.

(An extended, color version of this figure is available in the online journal.)

different broadband fluxes, e.g.,

$$
L_{\mathrm{TIR}}=\eta_{1} v L_{v}(25 \mu \mathrm{m})+\eta_{2} v L_{v}(60 \mu \mathrm{m})+\eta_{3} v L_{v}(100 \mu \mathrm{m})
$$

(IRAS-based),

$$
L_{\mathrm{TIR}}=\zeta_{1} v L_{v}(24 \mu \mathrm{m})+\zeta_{2} v L_{v}(70 \mu \mathrm{m})+\zeta_{3} v L_{v}(160 \mu \mathrm{m})
$$

(Spitzer-based),

$$
\begin{aligned}
L_{\mathrm{TIR}}= & \xi_{0} \nu L_{v}(8 \mu \mathrm{m})+\xi_{1} \nu L_{v}(24 \mu \mathrm{m})+\xi_{2} \nu L_{v}(70 \mu \mathrm{m}) \\
& +\xi_{3} \nu L_{v}(160 \mu \mathrm{m}) \quad(\text { Spitzer-based })
\end{aligned}
$$

where the coefficients are functions of the AGN fractional contribution to the 5-20 $\mu \mathrm{m}$ mid-infrared luminosity (for other recent formulations see also Boquien et al. 2010; Elbaz et al. 2011; Galametz et al. 2013). The coefficients are derived from a singular value decomposition solution to an overdetermined set of linear equations involving the individual broadband luminosities as well as the total infrared luminosity (TIR). Table 1 gives the various coefficients for a range of midinfrared AGN fractions and assuming rest-frame quantities. For the pure star-forming sequence (i.e., the top row of $0 \% \mathrm{AGN}$ ), the coefficients are similar to those already published in Dale \& Helou (2002).

The maximum error listed in Table 1 indicates the largest deviation of the TIR approximation, with the respect to the actual TIR, observed for the sequence of star-forming models parameterized by $\alpha_{\mathrm{SF}}$. The noticeably smaller maximum errors of the $24 / 70 / 160 \mu \mathrm{m}$ combination implies that this filter triplet does a better job of sampling the full infrared profile than the more wavelength-limited IRAS 25/60/100 $\mu \mathrm{m}$ combination; likewise, using the four 8/24/70/160 $\mu \mathrm{m}$ Spitzer fluxes better captures the full range of model variations than using just the three Spitzer/MIPS fluxes. The 70 and $160 \mu \mathrm{m}$ coefficients 


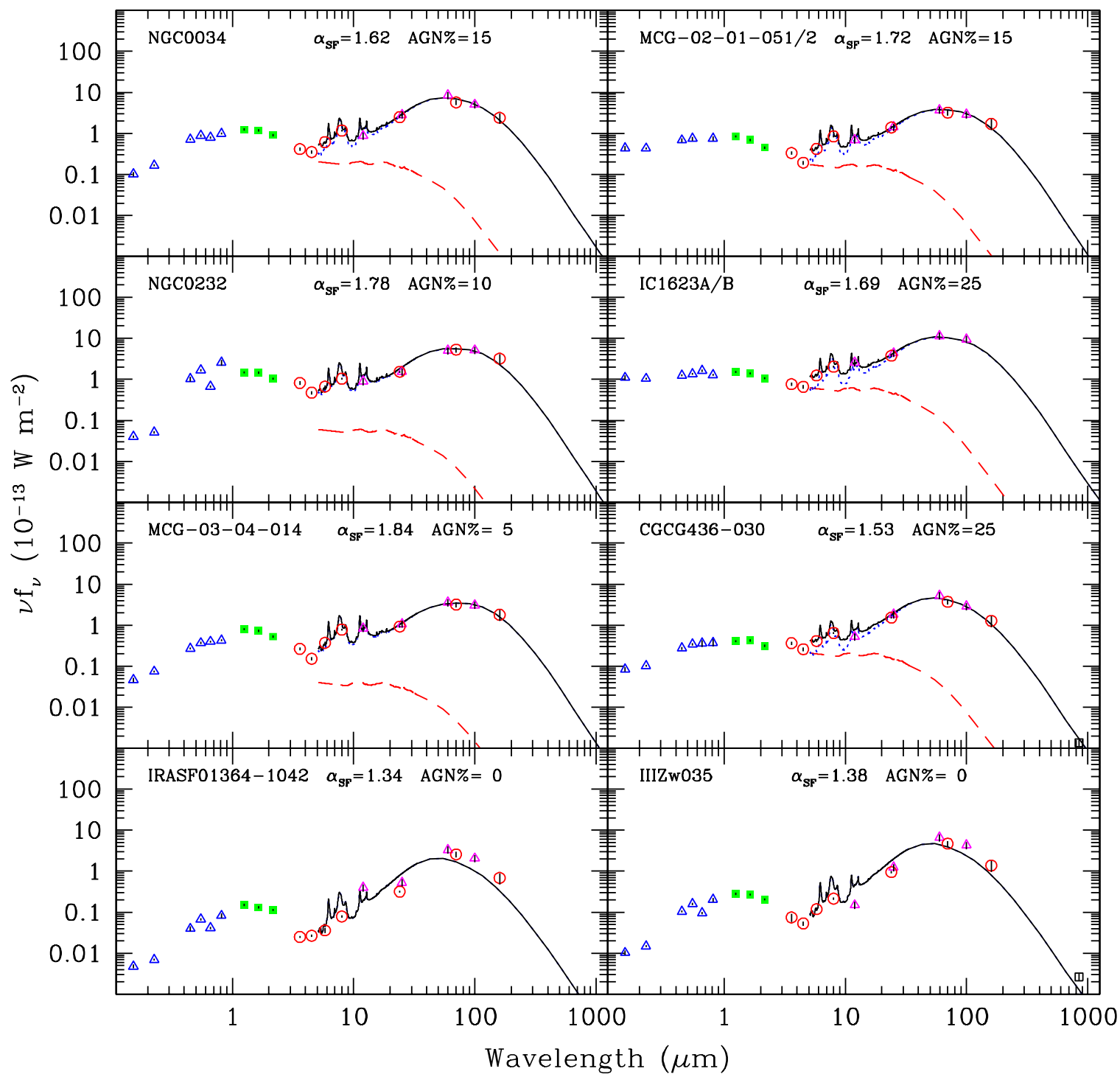

Figure 8. Globally integrated infrared/submillimeter spectral energy distributions for a subset of the GOALS sample of LIRGs and ULIRGs (Armus et al. 2009; U et al. 2012), sorted by right ascension. Open circles represent Spitzer data, open squares derive from the Two Micron All Sky Survey (2MASS), and open triangles stem from GALEX, SDSS, and IRAS. The dotted and dashed lines respectively trace the fitted star-forming and AGN components; the sum of the two components (solid line) is normalized to the Spitzer 24/70/160 $\mu \mathrm{m}$ and IRAS 25/60/100 $\mu \mathrm{m}$ data.

(An extended, color version of this figure is available in the online journal.)

in Equations (4) and (5) have formally been derived assuming Spitzer/MIPS bandpasses, but similar values are obtained for Herschel/PACS 70 and $160 \mu \mathrm{m}$ bandpasses.

Figure 10 shows how the 5MUSES sample is biased toward AGNs at higher infrared luminosities. Above a luminosity of $L($ TIR $) \sim 5 \times 10^{11} L_{\odot}$, nearly all 5MUSES sources with Spitzer and Herschel photometry are estimated to be dominated by AGNs. For reference, a similar evolution in AGN fraction with luminosity is seen for infrared-selected AKARI sources (see Figure 5 in Goto et al. 2011). The U et al. (2012) subset of the GOALS sample covers a much more limited range in luminosity and hence any trend is difficult to ascertain.

\section{SUMMARY}

A two-parameter family of infrared/submillimeter/radio SED models is presented. The first parameter governs the variety of long-wavelength spectral shapes observed for star-forming galaxies, whereas the second parameter quantifies the fractional contribution of AGN mid-infrared emission. The star-forming models are based on those presented in Dale \& Helou (2002) and incorporate updates at mid-infrared wavelengths using Spitzer spectral data. The AGN parameterization relies on the recent progress in generating panchromatic quasar databases. The particular spectrum adopted for this modeling effort is the median spectrum derived from a sample of Type 1 quasars (Shi et al. 2013). Because only two parameters are utilized, the fine-scale interpretive power of these models is necessarily limited, and they do not capture the full range of observed spectra. For example, optically thin emission is assumed and thus the models do not have the flexibility to account for the deep $9.7 \mu \mathrm{m}$ silicate absorption that can be evident in the spectra of many ULIRGs and quasars for which the accretion disks are viewed edge-on. Neither do the models capture the full range of observed starforming infrared spectra (e.g., SBS 0335-052 and NGC 1377; Houck et al. 2004; Roussel et al. 2006).

However, the models do a remarkable job in capturing the broad features seen in the infrared/submillimeter continuua of 


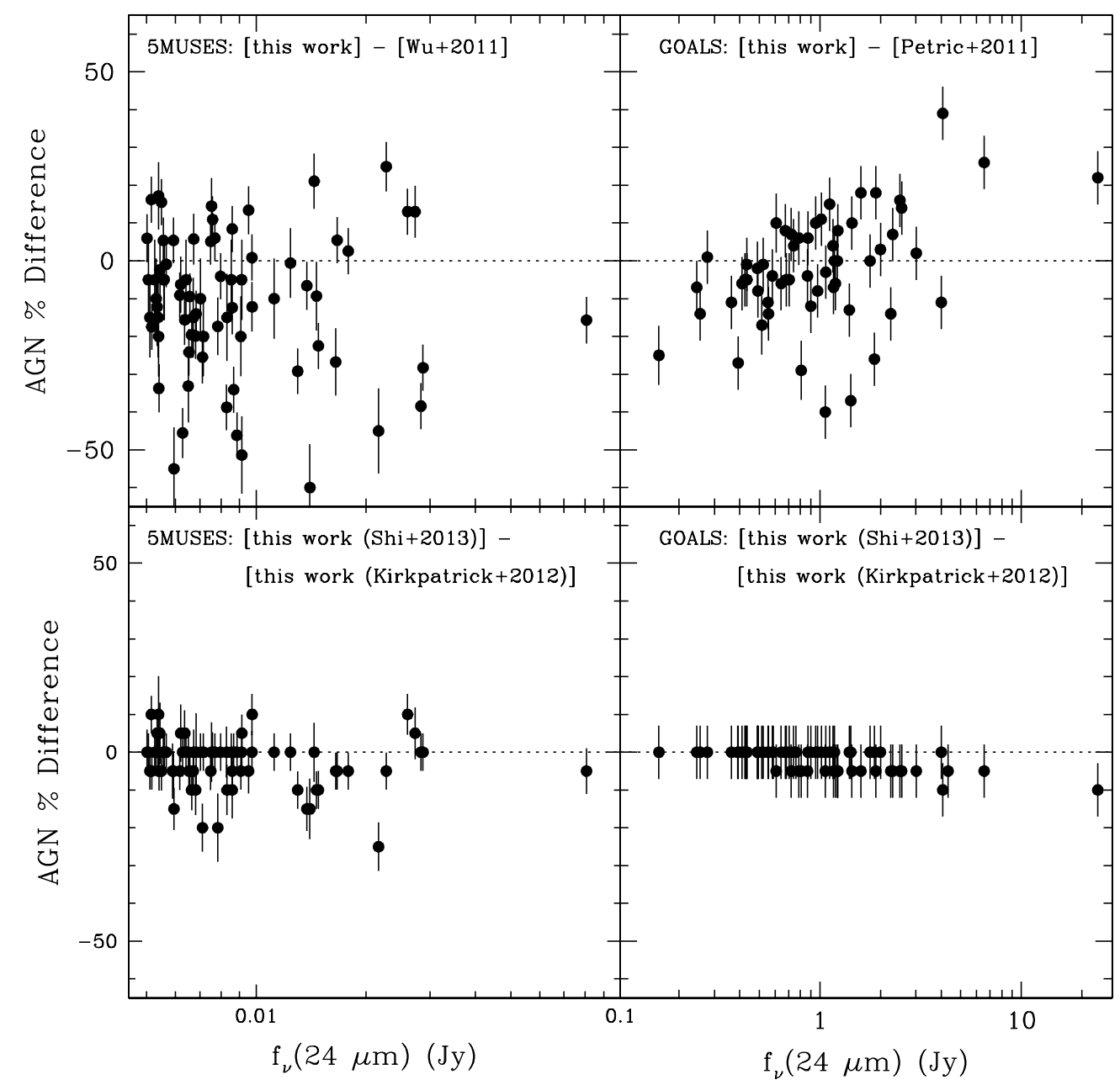

Figure 9. Comparisons of our mid-infrared AGN percentages using our infrared/submillimeter broadband SED-fitting approach and the Shi et al. (2013) quasar template with respect to those from (top left) the 5-35 $\mu \mathrm{m}$ continuum analysis of Wu et al. (2011); (top right) the EW(6.2 $\mu \mathrm{m})+6-15 \mu \mathrm{m}$ continuum analysis of Petric et al. (2011); (bottom) using our broadband SED-fitting approach coupled with the Kirkpatrick et al. (2012) AGN template. The error bars displayed in the top row derive from a sum in quadrature of the standard deviations in the Monte Carlo simulations described in Section 4.3 and the uncertainties from the literature fractional values (a 5\% uncertainty is assumed for $\mathrm{Wu}$ et al. 2011). The error bars displayed in the bottom row derive from a sum in quadrature of the standard deviations in the Monte Carlo simulations for both sets of fits.

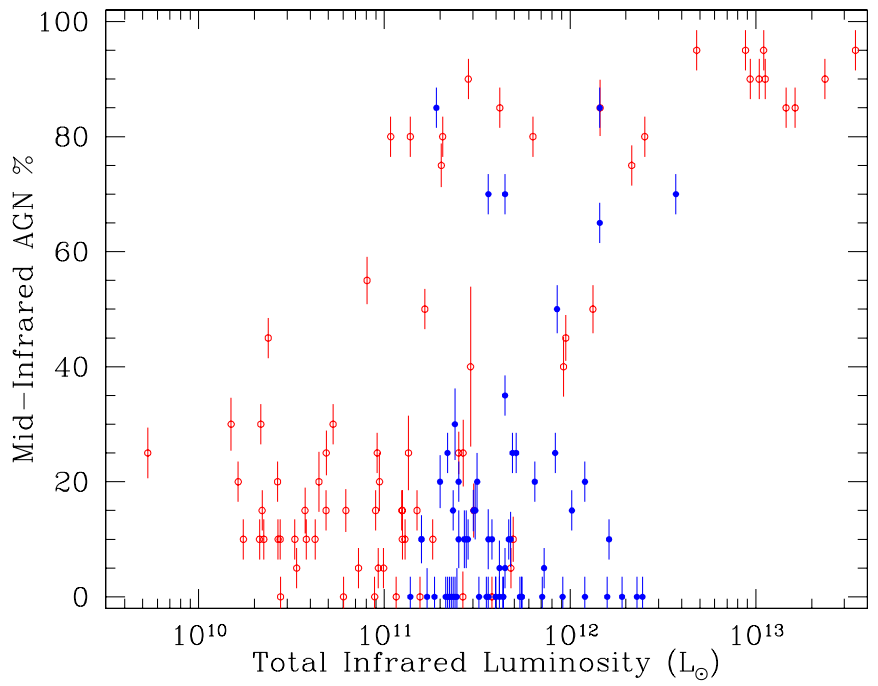

Figure 10. Our template-based 5MUSES (open circles) and GOALS (filled circles) AGN mid-infrared fractions as a function of infrared luminosity.

(A color version of this figure is available in the online journal.) multiple galaxy samples that span a large range in redshift, AGN activity, and infrared luminosity. Moreover, our estimates of the AGN fractional contributions to the mid-infrared luminosity are in most cases consistent with those previously published for these samples. We also show that the mid-infrared AGN fractional estimates are quite similar if an AGN template is adopted instead of a quasar spectrum. If only broadband infrared/submillimeter data are available and not continuum spectroscopy, these models can be used to statistically constrain the star-forming versus AGN properties of a large sample of galaxies (for a purely IRAS-based version, see also Figure 36(c) of Veilleux et al. 2009). But a detailed, case-by-case analysis of individual sources will of course require spectroscopic data (e.g., Murphy et al. 2009; Wu et al. 2011); the rich diversity of features available to mid-infrared spectroscopists (PAHs, continuum slope, emission line ratios, silicate absorption/emission) is necessary to more fully understand what drives the luminosity in many galaxies (Armus et al. 2007). Future efforts in developing "minimal" galaxy spectral models, i.e., those that rely on a small number of parameters, should focus on capturing the range of mid-infrared spectral slopes and emission and absorption features evident in galaxy and AGN spectra. 
Table 1

Coefficients for Determining 5-1100 $\mu \mathrm{m}$ Total Infrared Luminosity

\begin{tabular}{|c|c|c|c|c|c|c|c|c|c|c|c|c|c|}
\hline $\begin{array}{l}\text { AGN } \\
\text { Fraction } \\
(\%)\end{array}$ & $\eta_{1}$ & $\eta_{2}$ & $\eta_{3}$ & $\begin{array}{c}\text { Max } \\
\text { Error } \\
(\%)\end{array}$ & $\zeta_{1}$ & $\zeta_{2}$ & $\zeta_{3}$ & $\begin{array}{c}\text { Max } \\
\text { Error } \\
(\%)\end{array}$ & $\xi_{0}$ & $\xi_{1}$ & $\xi_{3}$ & $\xi_{3}$ & $\begin{array}{c}\text { Max } \\
\text { Error } \\
(\%)\end{array}$ \\
\hline 00 & 2.333 & -0.196 & 1.566 & +6.7 & 1.548 & 0.767 & 1.285 & +0.2 & -0.173 & 1.541 & 0.766 & 1.368 & -0.03 \\
\hline 05 & 2.345 & -0.203 & 1.570 & +6.5 & 1.558 & 0.763 & 1.299 & +0.1 & -0.052 & 1.556 & 0.763 & 1.325 & -0.01 \\
\hline 10 & 2.343 & -0.202 & 1.570 & +6.5 & 1.559 & 0.764 & 1.309 & +0.1 & -0.043 & 1.558 & 0.764 & 1.331 & +0.03 \\
\hline 15 & 2.328 & -0.193 & 1.566 & +6.4 & 1.558 & 0.767 & 1.318 & +0.1 & -0.210 & 1.554 & 0.766 & 1.430 & -0.12 \\
\hline 20 & 2.345 & -0.204 & 1.572 & +6.2 & 1.566 & 0.765 & 1.335 & +0.1 & -0.041 & 1.566 & 0.764 & 1.358 & -0.01 \\
\hline 25 & 2.349 & -0.206 & 1.574 & +6.0 & 1.574 & 0.763 & 1.352 & -0.1 & +0.087 & 1.574 & 0.764 & 1.302 & +0.05 \\
\hline 30 & 2.352 & -0.207 & 1.575 & +5.9 & 1.579 & 0.763 & 1.369 & -0.1 & +0.028 & 1.579 & 0.763 & 1.352 & -0.01 \\
\hline 35 & 2.359 & -0.212 & 1.578 & +5.7 & 1.588 & 0.761 & 1.391 & -0.1 & +0.141 & 1.586 & 0.762 & 1.301 & +0.05 \\
\hline 40 & 2.366 & -0.216 & 1.580 & +5.5 & 1.603 & 0.756 & 1.419 & -0.3 & +0.323 & 1.594 & 0.759 & 1.203 & +0.13 \\
\hline 45 & 2.356 & -0.210 & 1.579 & +5.4 & 1.606 & 0.759 & 1.440 & -0.2 & +0.101 & 1.602 & 0.761 & 1.368 & -0.05 \\
\hline 50 & 2.366 & -0.217 & 1.584 & +5.1 & 1.619 & 0.756 & 1.475 & -0.3 & +0.279 & 1.603 & 0.761 & 1.264 & +0.06 \\
\hline 55 & 2.381 & -0.225 & 1.586 & +4.8 & 1.633 & 0.755 & 1.511 & -0.3 & +0.250 & 1.613 & 0.759 & 1.307 & +0.03 \\
\hline 60 & 2.373 & -0.220 & 1.587 & +4.6 & 1.643 & 0.757 & 1.554 & -0.3 & +0.214 & 1.621 & 0.761 & 1.366 & -0.04 \\
\hline 65 & 2.382 & -0.225 & 1.589 & +4.2 & 1.671 & 0.749 & 1.611 & -0.5 & +0.355 & 1.625 & 0.757 & 1.270 & +0.04 \\
\hline 70 & 2.387 & -0.228 & 1.591 & +3.9 & 1.691 & 0.749 & 1.679 & -0.5 & +0.364 & 1.631 & 0.758 & 1.292 & +0.02 \\
\hline 75 & 2.390 & -0.230 & 1.593 & +3.4 & 1.724 & 0.745 & 1.767 & -0.6 & +0.454 & 1.628 & 0.759 & 1.225 & +0.07 \\
\hline 80 & 2.391 & -0.232 & 1.597 & +3.0 & 1.754 & 0.749 & 1.884 & -0.5 & +0.361 & 1.656 & 0.762 & 1.391 & -0.02 \\
\hline 85 & 2.407 & -0.240 & 1.594 & +2.4 & 1.827 & 0.731 & 2.048 & -0.7 & +0.565 & 1.623 & 0.758 & 1.136 & +0.10 \\
\hline 90 & 2.400 & -0.238 & 1.605 & +1.8 & 1.908 & 0.727 & 2.296 & -0.6 & +0.503 & 1.662 & 0.757 & 1.299 & +0.02 \\
\hline 95 & 2.412 & -0.244 & 1.594 & +0.9 & 2.053 & 0.712 & 2.714 & -0.5 & +0.578 & 1.644 & 0.760 & 1.208 & +0.03 \\
\hline
\end{tabular}

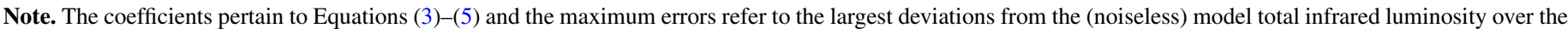
range of $\alpha_{\mathrm{SF}}$.

We appreciate helpful discussions with Adam Myers, Mike Brotherton, Zhaohui Shang, Allison Kirkpatrick, Emeric Le Floc'h, and James Mullaney. Support for this work, part of the Spitzer Space Telescope Legacy Science Program 40539, was provided by NASA and issued by the Jet Propulsion Laboratory, California Institute of Technology under NASA contract 1407. Herschel is an ESA space observatory with science instruments provided by European-led Principal Investigator consortia and with important participation from NASA. This research has made use of the NASA/IPAC Infrared Science Archive, which is operated by the Jet Propulsion Laboratory, California Institute of Technology, under contract with NASA. We gratefully acknowledge NASA's support for the construction, operation, and science analysis for the Galaxy Evolution Explorer $(G A L E X)$ mission, developed in cooperation with the Centre National d'Etudes Spatiales of France and the Korean Ministry of Science and Technology. Funding for the Sloan Digital Sky Survey (SDSS) and SDSS-II has been provided by the Alfred P. Sloan Foundation, the Participating Institutions, the NSF, the U.S. Department of Energy, NASA, the Japanese Monbukagakusho, the Max Planck Society, and the Higher Education Funding Council for England. This publication makes use of data products from the Two Micron All Sky Survey, which is a joint project of the University of Massachusetts and the Infrared Processing and Analysis Center/California Institute of Technology, funded by the National Aeronautics and Space Administration and the National Science Foundation.

\section{REFERENCES}

Armus, L., Charmandaris, V., Bernard-Salas, J., et al. 2007, ApJ, 656, 148 Armus, L., Mazzarella, J. M., Evans, A. S., et al. 2009, PASP, 121, 559

Baes, M., Clemens, M., Xilouris, E. M., et al. 2010, A\&A, 518, 53

Berta, S., Lutz, D., Santini, P., et al. 2013, A\&A, 551, 100

Boquien, M., Bendo, G., Calzetti, D., et al. 2010, ApJ, 713, 626

Chapman, S. C., Helou, G., Lewis, G. F., \& Dale, D. A. 2003, ApJ, 588, 186

Chary, R., \& Elbaz, D. 2001, ApJ, 556, 562 da Cunha, E., Charlot, S., \& Elbaz, D. 2008, MNRAS, 388, 1595

Dale, D. A., Aniano, G., Engelbracht, C. W., et al. 2012, ApJ, 745, 95

Dale, D. A., \& Helou 2002, ApJ, 576, 159

Dale, D. A., Helou, G., Contursi, A., Silbermann, N. A., \& Kolhatkar, S. 2001, ApJ, 549, 215

Dale, D. A., Silbermann, N. A., Helou, G., et al. 2000, AJ, 120, 583

Dale, D. A., Smith, J. D. T., Schlawin, E. A., et al. 2009, ApJ, 693, 1821

Del Moro, A., Alexander, D. M., Mullaney, J. R., et al. 2013, A\&A, 549, 59

Désert, F. X., Boulanger, F., \& Puget, J. L. 1990, A\&A, 237, 215

Diamond-Stanic, A. M., \& Rieke, G. H. 2010, ApJ, 724, 140

Draine, B. T., \& Li, A. 2007, ApJ, 657, 810

Elbaz, D., Dickinson, M., Hwang, H. S., et al. 2011, A\&A, 533, A119

Elvis, M., Wilkes, B. J., McDowell, J. C., et al. 1994, ApJS, 95, 1

Fadda, D., Marleau, F. R., Storrie-Lombardi, L. J., et al. 2006, AJ, 131, 2859

Fan, X., Strauss, M. A., Schneider, D. P., et al. 2001, AJ, 121, 54

Frayer, D. T., Fadda, D., Yan, L., et al. 2006, AJ, 131, 250

Fu, H., Yan, L., Scoville, N. Z., et al. 2010, ApJ, 722, 653

Galametz, M., Kennicutt, R. C., Calzetti, D., et al. 2013, MNRAS, 431, 1956

Galliano, F., Dwek, E., \& Chanial, P. 2008, ApJ, 672, 214

Genzel, R., Lutz, D., Sturm, E., et al. 1998, ApJ, 498, 579

Gordon, K. D., Misselt, K. A., Witt, A. N., \& Clayton, G. C. 2001, ApJ, 551,269

Goto, T., Arnouts, S., Malkan, M., et al. 2011, MNRAS, 414, 1903

Goto, T., Takagi, T., Matsuhara, H., et al. 2010, A\&A, 514, 6

Groves, B., Dopita, M. A., Sutherland, R. S., et al. 2008, ApJS, 176, 438

Hao, L., Spoon, H. W. W., Sloan, G. C., et al. 2005, ApJL, 625, L75

Hermelo, I., Lisenfeld, U., Relaño, M., et al. 2013, A\&A, 549, 70

Hernán-Caballero, A., Pérez-Fournon, I., Hatziminaoglou, E., et al. 2009, MNRAS, 395, 1695

Houck, J. R., Charmandaris, V., Brandl, B. R., et al. 2004, ApJS, 154, 211

Kirkpatrick, A., Calzetti, D., Galametz, M., et al. 2013, ApJ, 778, 51

Kirkpatrick, A., Pope, A., Alexander, D. M., et al. 2012, ApJ, 759, 139

Lacy, M., Storrie-Lombardi, L. J., Sajina, A., et al. 2004, ApJS, 154, 166

Laurent, O., Mirabel, I. F., Charmandaris, V., et al. 2000, A\&A, 359, 887

Lonsdale, C. J., Smith, H. E., Rowan-Robinson, M., et al. 2003, PASP, 115, 897

Lu, N., Helou, G., Werner, M. W., et al. 2003, ApJ, 588, 199

Madden, S. C., Galliano, F., Jones, A. P., \& Sauvage, M. 2006, A\&A, 446, 877

Magdis, G. E., Rigopoulou, D., Helou, G., et al. 2013, A\&A, 558, 136

Mendez, A. J., Coil, A. L., Aird, J., et al. 2013, ApJ, 770, 40

Moustakas, J., Kennicutt, R. C., Jr., Tremonti, C. A., et al. 2010, ApJS, 190, 233

Mullaney, J. R., Alexander, D. M., Goulding, A. D., \& Hickox, R. C. 2011, MNRAS, 414, 1082

Murphy, E. J., Chary, R.-R., Alexander, D. M., et al. 2009, ApJ, 698, 1380 
Oliver, S. J., Wang, L., Smith, A. J., et al. 2010, A\&A, 518, 21

Peeters, E., Spoon, H. W. W., \& Tielens, A. G. G. M. 2004, ApJ, 613, 986

Petric, A. O., Armus, L., Howell, J., et al. 2011, ApJ, 730, 28

Popescu, C. C., Misiriotis, A., Kylafis, N. D., Tuffs, R. J., \& Fischera, J. 2000 A\&A, 362, 138

Richards, G. T., Lacy, M., Storrie-Lombardi, L. J., et al. 2006, ApJS, 166, 470

Rieke, G. H., Alonso-Herrero, A., Weiner, B. J., et al. 2009, ApJ, 692, 556

Roussel, H., Helou, G., Smith, J. D., et al. 2006, ApJ, 646, 841

Schartmann, M., Meiseheimer, K., Camenzind, M., et al. 2008, A\&A, 482, 67

Shang, Z., Brotherton, M. S., Wills, B. J., et al. 2011, ApJS, 196, 2

Shi, Y., Helou, G., Armus, L., Stierwalt, S., \& Dale, D. A. 2013, ApJ, 764, 28
Siebenmorgen, R., \& Krügel, E. 2007, A\&A, 461, 445

Silva, L., Granato, G. L., Bressan, A., \& Danese, L. 1998, ApJ, 509, 103

Smith, J. D. T., Draine, B. T., Dale, D. A., et al. 2007, ApJ, 656, 770

Spoon, H. W. W., Marshall, J. A., Houck, J. R., et al. 2007, ApJL, 654, L49

Stern, D., Eisenhardt, P., Gorjian, V., et al. 2005, ApJ, 631, 163

Stierwalt, S., Armus, L., Surace, J. A., et al. 2013, ApJS, 206,

U, V., Sanders, D. B., Mazzarella, J. M., et al. 2012, ApJS, 203, 9

Veilleux, S., Rupke, D. S. N., Kim, D.-C., et al. 2009, ApJS, 182, 628

Wu, Y., Helou, G., Armus, L., et al. 2010, ApJ, 723, 895

Wu, Y., Shi, Y., Helou, G., et al. 2011, ApJ, 734, 40

Yan, L., Donoso, E., Tsai, C.-W., et al. 2013, AJ, 145, 55 